Geometry $\&$ Topology

Volume 6 (2002) 541-562

Published: 24 November 2002

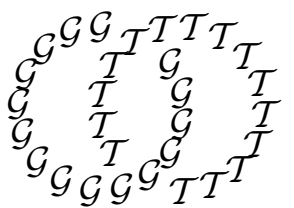

\title{
Virtual Betti numbers of genus 2 bundles
}

\author{
Joseph D MAsters \\ Mathematics Department, Rice University \\ Houston, TX 77005-1892, USA \\ Email: mastersj@math.rice.edu
}

\begin{abstract}
We show that if $M$ is a surface bundle over $S^{1}$ with fiber of genus 2, then for any integer $n, M$ has a finite cover $\widetilde{M}$ with $b_{1}(\widetilde{M})>n$. A corollary is that $M$ can be geometrized using only the "non-fiber" case of Thurston's Geometrization Theorem for Haken manifolds.
\end{abstract}

\section{AMS Classification numbers Primary: 57M10}

Secondary: 57R10

Keywords: 3-manifold, geometrization, virtual Betti number, genus 2 surface bundle

Proposed: Walter Neumann

Seconded: Cameron Gordon, Joan Birman
Received: 15 January 2002

Revised: 9 August 2002

(c) Geometry $\mathcal{G}$ Topology $\mathcal{P}$ ublications 


\section{Introduction}

Let $M$ be a 3-manifold. Define the virtual first Betti number of $M$ by the formula $v b_{1}(M)=\sup \left\{b_{1}(\widetilde{M}): \widetilde{M}\right.$ is a finite cover of $\left.\mathrm{M}\right\}$.

The following well-known conjecture is a strengthening of Waldhausen's conjecture about virtually Haken 3-manifolds.

Conjecture 1.1 Let $M$ be a closed irreducible 3-manifold with infinite fundamental group. Then either $\pi_{1} M$ is virtually solvable, or $v b_{1}(M)=\infty$.

Combining the Seifert Fiber Space Theorem, the Torus Theorem, and arguments involving characteristic submanifolds, Conjecture 1.1 is known to be true in the case that $\pi_{1} M$ contains a subgroup isomorphic to $\mathbb{Z} \times \mathbb{Z}$. However, little is known in the atoroidal case.

In [3], Gabai called attention to Conjecture 1.1 in the case that $M$ fibers over $S^{1}$. This seems a natural place to start, in light of Thurston's conjecture that every closed hyperbolic 3-manifold is finitely covered by a bundle. The purpose of this paper is to give some affirmative results for this case. In particular, we prove Conjecture 1.1 in the case where $M$ is a genus 2 bundle.

Throughout this paper, if $f: F \rightarrow F$ is an automorphism of a surface, then $M_{f}$ denotes the associated mapping torus. Our main theorem is the following:

Theorem 1.2 Let $f: F \rightarrow F$ be an automorphism of a surface. Suppose there is a finite group $G$ of automorphisms of $F$, so that $f$ commutes with each element of $G$, and $F / G$ is a torus with at least one cone point. Then $v b_{1}\left(M_{f}\right)=\infty$.

We have the following corollaries:

Corollary 1.3 Suppose $F$ has genus at least 2, and $f: F \rightarrow F$ is an automorphism which commutes with a hyper-elliptic involution on $F$. Then $v b_{1}\left(M_{f}\right)=\infty$.

Proof Let $\tau$ be the hyper-elliptic involution. Since $f$ commutes with $\tau, f$ induces an automorphism $\bar{f}$ of $F / \tau$, which is a sphere with $2 g+2$ order 2 cone points. $F / \tau$ is double covered by a hyperbolic orbifold $T$, whose underlying space is a torus. By passing to cyclic covers of $M$, we may replace $f$ (and $\bar{f}$ ) with powers, and so we may assume $\bar{f}$ lifts to $T$. Corresponding to $T$, there is a 2 -fold cover $\widetilde{F}$ of $F$ to which $f$ lifts, and an associated cover $\widetilde{M}$ of $M$ whose monodromy satisfies the hypotheses of Theorem 1.2. 
Corollary 1.4 Let $M$ be a surface bundle with fiber $F$ of genus 2. Then $v b_{1}(M)=\infty$.

Proof Since the fiber has genus 2, the monodromy map commutes (up to isotopy) with the central hyper-elliptic involution on $F$. The result now follows from Corollary 1.3.

To state our next theorem, we require some notation. Recall that, by [4], the mapping class group of a surface is generated by Dehn twists in the loops pictured in Figure 1. If $\ell$ is a loop in a surface, we let $D_{\ell}$ denote the righthanded Dehn twist in $\ell$.

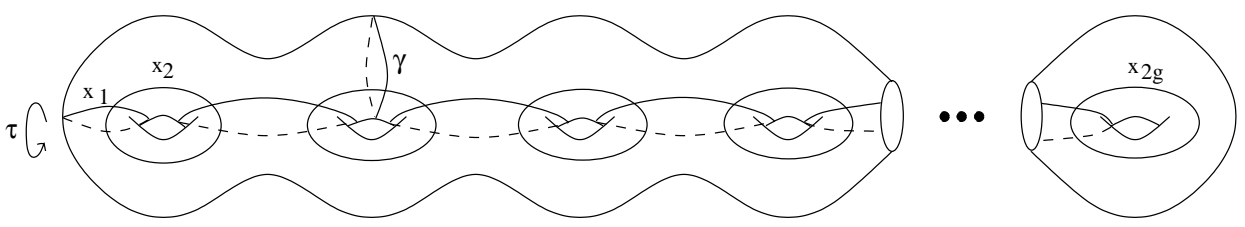

Figure 1: The mapping class group is generated by Dehn twists in these loops.

With the exception of $D_{\gamma}$, these Dehn twists each commute with the involution $\tau$ pictured in Figure 1. Let $H$ be the subgroup of the mapping class group generated by the $D_{x_{i}}$ 's. For any monodromy $f \in H$, we may apply Corollary 1.3 to show that the associated bundle $M$ has $v b_{1}(M)=\infty$. The proof provides an explicit construction of covers- a construction which may be applied to any bundle, regardless of monodromy. These covers will often have extra homology, even when the monodromy does not commute with $\tau$. For example, we have the following theorem, which is proved in Section 7 .

Theorem 1.5 Let $M$ be a surface bundle over $S^{1}$ with fiber $F$ and monodromy $f: F \rightarrow F$. Suppose that $f$ lies in the subgroup of the mapping class group generated by $D_{x_{1}}, \ldots, D_{x_{2 g}}$ and $D_{\gamma}^{8}$. Then $v b_{1}(M)=\infty$.

None of the proofs makes any use of a geometric structure. In fact, for a bundle satisfying the hypotheses of one of the above theorems, we may give an alternative proof of Thurston's hyperbolization theorem for fibered 3-manifolds. For example, we have:

Theorem 1.6 (Thurston) Let $M$ be an atoroidal surface bundle over $S^{1}$ with fiber a closed surface of genus 2. Then $M$ is hyperbolic. 
Proof By Corollary 1.4, $M$ has a finite cover $\widetilde{M}$ with $b_{1}(\widetilde{M}) \geq 2$. Therefore, by [12], $\widetilde{M}$ contains a non-separating incompressible surface which is not a fiber in a fibration. Now the techniques of the non-fiber case of Thurston's Geometrization Theorem (see [8]) may be applied to show that $\widetilde{M}$ is hyperbolic. Since $M$ has a finite cover which is hyperbolic, the Mostow Rigidity Theorem implies that $M$ is homotopy equivalent to a hyperbolic $3-$ manifold. Since $M$ is Haken, Waldhausen's Theorem ([13]) implies that $M$ is in fact homeomorphic to a hyperbolic 3-manifold.

We say that a surface automorphism $f: F \rightarrow F$ is hyper-elliptic if it commutes with some hyperelliptic involution on $F$. Corollary 1.3 prompts the question: is a hyper-elliptic monodromy always attainable in a finite cover? Our final theorem shows that the answer is no.

Theorem 1.7 There exists a closed surface $F$, and a pseudo-Anosov automorphism $f: F \rightarrow F$, such that $f$ does not lift to become hyper-elliptic in any finite cover of $F$.

The proof of Theorem 1.7 will be given in Section 8 .

Acknowledgements I would like to thank Andrew Brunner, Walter Neumann and Hyam Rubinstein, whose work suggested the relevance of punctured tori to this problem. I also thank Mark Baker, Darren Long, Alan Reid and the referee for carefully reading previous versions of this paper, and providing many helpful comments. Alan Reid also helped with the proof of Theorem 1.7. Thanks also to The University of California at Santa Barbara, where this work was begun.

This research was supported by an NSF Postdoctoral Fellowship.

\section{Homology of bundles: generalities}

In what follows, we shall try to keep notation to a minimum; in particular we shall often neglect to distinguish notationally between the monodromy map $f$, and the various maps which $f$ induces on covering spaces or projections. All homology groups will be taken with $\mathbb{Q}$ coefficents.

Suppose $f$ is an automorphism of a closed 2-orbifold $O$. The mapping torus $M_{f}$ associated with $O$ is a 3 -orbifold, whose singular set is a link. We have the following well-known formula for the first Betti number of $M_{f}$ :

$$
b_{1}\left(M_{f}\right)=1+\operatorname{dim}\left(\operatorname{fix}\left(f_{*}\right)\right) \text {, }
$$


where $\operatorname{fix}\left(f_{*}\right)$ is the subspace of $H_{1}(O)$ on which $f_{*}$ acts trivially. This can be derived by abelianizing the standard HNN presentation for $\pi_{1} M_{f}$.

Suppose now that $O$ is obtained from a punctured surface $F$ by filling in the punctures with disks or cone points, and suppose that $f$ restricts to an automorphism of $F$. Let $V \subset H_{1}(F, \partial F)$ be the subspace on which the induced map $f_{*}$ acts trivially. Then the first Betti number for the mapping torus of $O$ can also be computed by the following formula.

Proposition 2.1 For $M_{f}$ and $V$ as above, we have $b_{1}\left(M_{f}\right)=1+\operatorname{dim}(V)$.

Proof By Formula $1, b_{1}\left(M_{f}\right)=1+\operatorname{dim}(W)$, where $W \subset H_{1}(O)$ is the subspace on which $f_{*}$ acts trivially.

Let $i: F \rightarrow O$ be the inclusion map, and let $K$ be the kernel of the quotient map from $H_{1}(F)$ onto $H_{1}(F, \partial F)$. The cone-point relations imply that every element in $i_{*} K$ is a torsion element in $H_{1}(O)$; since we are using $\mathbb{Q}$-coefficients, $i_{*} K$ is in fact trivial in $H_{1}(O)$. The action of $f_{*}$ on $H_{1}(O)$ is therefore identical to the action of $f_{*}$ on $H_{1}(F, \partial F)$, so $\operatorname{dim}(W)=\operatorname{dim}(V)$, which proves the formula.

We will also need the following technical proposition.

Proposition 2.2 Let $F$ be a punctured surface, and let $f: F \rightarrow F$ be an automorphism which fixes the punctures. Let $F^{+}$be a surface obtained from $F$ by filling in one or more of the punctures, and let $f^{+}: F^{+} \rightarrow F^{+}$be the map induced by $f$. Suppose $\widetilde{F^{+}}$is a cover of $F^{+}$, such that $f^{+}$lifts, and suppose $\alpha^{+} \in \widetilde{F^{+}}$is a loop which misses all filled-in punctures, and such that $f^{+}\left[\alpha^{+}\right]=\left[\alpha^{+}\right] \in H_{1}\left(\widetilde{F^{+}}, \partial \widetilde{F^{+}}\right)$. Let $\widetilde{F}$ be the cover of $F$ corresponding to the cover $\widetilde{F^{+}}$of $F^{+}$, and let $i: \widetilde{F} \rightarrow \widetilde{F^{+}}$be the natural inclusion map. Let $\alpha=i^{-1} \alpha^{+}$. Then $f[\alpha]=[\alpha] \in H_{1}(\widetilde{F}, \partial \widetilde{F})$.

Proof The surface $\widetilde{F^{+}}$is obtained from $\widetilde{F}$ by filling in a certain number of punctures, say $\beta_{1}, \ldots, \beta_{k}$, of $\widetilde{F}$. The map $f: H_{1}(\widetilde{F}, \partial \widetilde{F}) \rightarrow H_{1}(\widetilde{F}, \partial \widetilde{F})$ may be obtained from the map $f: \pi_{1} \widetilde{F} \rightarrow \pi_{1} \widetilde{F}$ by:

(1) First add the relations $\beta_{1}, \ldots, \beta_{k}=i d$. There is an induced map

$$
f: \pi_{1} \widetilde{F} /<\beta_{1}=\ldots=\beta_{k}=i d>\rightarrow \pi_{1} \widetilde{F} /<\beta_{1}=\ldots=\beta_{k}=i d>.
$$

(2) Add the relations which kill the remaining boundary components. 
(3) Add the relations $[x, y]=i d$ for all $x, y \in \pi_{1} \widetilde{F^{+}}$.

After completing step 1 , one has precisely the action of $f$ on $\pi_{1} \widetilde{F^{+}}$. After completing steps 2 and 3 , one then has the action of $f$ on $H_{1}\left(\widetilde{F^{+}}, \partial \widetilde{F^{+}}\right)$. So the action of $f$ on these groups is identical, and $[\alpha]$ is a fixed class.

If $\Gamma$ is a group, we may define $b_{1}(\Gamma)$ to be the $\mathbb{Q}$-rank of its abelianization, and the virtual first Betti number of $\Gamma$ by

$$
v b_{1}(\Gamma)=\sup \left\{b_{1}(\widetilde{\Gamma}): \widetilde{\Gamma} \text { is a finite index subgroup of } \Gamma\right\} .
$$

Clearly, for a 3-manifold $M, v b_{1}(M)=v b_{1}\left(\pi_{1}(M)\right)$. We have the following:

Lemma 2.3 Suppose $\Gamma$ maps onto a group $\Delta$. Then

$$
\left(v b_{1}(\Gamma)-b_{1}(\Gamma)\right) \geq\left(v b_{1}(\Delta)-b_{1}(\Delta)\right) .
$$

Before proving this, we will need a preliminary lemma. We let $H_{1}(\Gamma)$ denote the abelianization of $\Gamma$, tensored over $\mathbb{Q}$. Representing $\Gamma$ by a $2-$ complex $C_{\Gamma}$, then $H_{1}(\Gamma) \cong H_{1}\left(C_{\Gamma}\right)$.

Any subgroup $\widetilde{\Gamma}$ of $\Gamma$ determines a $2-$ complex $\widetilde{C_{\Gamma}}$ and a covering map $p$ : $\widetilde{C_{\Gamma}} \rightarrow$ $C_{\Gamma}$. We can define a map $j: H_{1}\left(C_{\Gamma}\right) \rightarrow H_{1}\left(\widetilde{C_{\Gamma}}\right)$ by the rule $j([\ell])=\left[p^{-1} \ell\right]$, for any loop $\ell$ in $C_{\Gamma}$. If $\ell$ bounds a $2-$ chain in $C_{\Gamma}$, then $p^{-1} \ell$ bounds a 2 -chain in $\widetilde{C_{\Gamma}}$, so this map is well-defined. Using the isomorphisms between the homology of the groups and the homology of the 2-complexes, we get a map, which we also call $j$, from $H_{1}(\Gamma)$ to $H_{1}(\widetilde{\Gamma})$.

Lemma 2.4 If $\widetilde{\Gamma}$ has finite index, then the map $j$ is injective.

Proof Suppose $[\gamma] \in \operatorname{Ker}(j)$, where $\gamma$ is an element of $\Gamma$, and let $\ell \in C_{\Gamma}$ be a corresponding loop. Then $[\ell] \in \operatorname{Ker}(j)$, so $\left[p^{-1} \ell\right]=0$, and therefore

$$
0=p_{*}\left[p^{-1} \ell\right]=n[\ell],
$$

where $n$ is the index of $\widetilde{\Gamma}$. Since we are using $\mathbb{Q}$-coefficients, $H_{1}\left(C_{\Gamma}\right)$ is torsionfree, so $[\ell]=0$ in $H_{1}\left(C_{\Gamma}\right)$, and therefore $[\gamma]=0$ in $H_{1}(\Gamma)$.

Proof of Lemma 2.3 Let $f: \Gamma \rightarrow \Delta$ be a surjective map. We have the following commutative diagram:

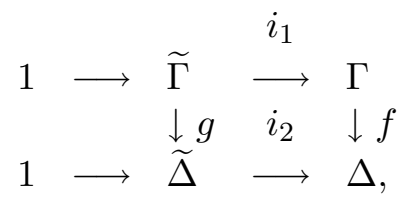


where $i_{1}$ and $i_{2}$ are inclusion maps, and the surjective map $g$ is induced from the other maps. There is an induced diagram on the homology:

$$
\begin{array}{ccc} 
& i_{1 *} & \\
H_{1}(\widetilde{\Gamma}) & \longrightarrow & H_{1}(\Gamma) \\
\downarrow g_{*} & i_{2 *} & \downarrow f_{*} \\
H_{1}(\widetilde{\Delta}) & \longrightarrow & H_{1}(\Delta) .
\end{array}
$$

Let $j_{1}: H_{1}(\Gamma) \longrightarrow H_{1}(\widetilde{\Gamma})$ and $j_{2}: H_{1}(\Delta) \longrightarrow H_{1}(\widetilde{\Delta})$ be the injective maps given by Lemma 2.4. These maps give rise to the following diagram, which can be checked to be commutative:

$$
\begin{array}{ccccc} 
& j_{1} & & & \\
H_{1}(\widetilde{\Gamma}) & \longleftarrow & H_{1}(\Gamma) & \longleftarrow & 0 \\
\downarrow g_{*} & j_{2} & \downarrow f_{*} & & \\
H_{1}(\widetilde{\Delta}) & \longleftarrow & H_{1}(\Delta) & \longleftarrow & 0 .
\end{array}
$$

The definitions of the maps give that

$$
i_{1 *} j_{1}([\gamma])=n[\gamma],
$$

and a similar relation for $i_{2 *}$ and $j_{2}$. Therefore $\operatorname{Ker}\left(i_{1 *}\right)$ and Image $\left(j_{1}\right)$ are disjoint subspaces of $H_{1}(\widetilde{\Gamma})$. Also,

$$
\begin{aligned}
\operatorname{dim}\left(H_{1}(\widetilde{\Gamma})\right) & =\operatorname{dim}\left(\operatorname{Ker}\left(i_{1 *}\right)\right)+\operatorname{dim}\left(\operatorname{Image}\left(i_{1 *}\right)\right) \\
& =\operatorname{dim}\left(\operatorname{Ker}\left(i_{1 *}\right)\right)+\operatorname{dim}\left(H_{1}(\Gamma)\right), \text { by the relation }\left(^{*}\right) \\
& =\operatorname{dim}\left(\operatorname{Ker}\left(i_{1 *}\right)\right)+\operatorname{dim}\left(\operatorname{Image}\left(j_{1}\right)\right),
\end{aligned}
$$

so we get $H_{1}(\widetilde{\Gamma})=\operatorname{Ker}\left(i_{1 *}\right) \times \operatorname{Image}\left(j_{1}\right)$, and similarly $H_{1}(\widetilde{\Delta})=\operatorname{Ker}\left(i_{2 *}\right) \times$ Image $\left(j_{2}\right)$. Substituting these decompositions into the previous diagram gives:

$$
\begin{aligned}
& \operatorname{Ker}\left(i_{1 *}\right) \times \operatorname{Image}\left(j_{1}\right) \stackrel{j_{1}}{\longleftarrow} H_{1}(\Gamma) \longleftarrow 0 \\
& \downarrow g_{*} \quad j_{2} \quad \downarrow f_{*} \\
& \operatorname{Ker}\left(i_{2 *}\right) \times \operatorname{Image}\left(j_{2}\right) \longleftarrow H_{1}(\Delta) \longleftarrow 0 .
\end{aligned}
$$

By the commutativity of this diagram, we have that $g_{*} \operatorname{Image}\left(j_{1}\right) \subset \operatorname{Image}\left(j_{2}\right)$. Also, by the commutativity of a previous diagram, we have $g_{*} \operatorname{Ker}\left(i_{1 *}\right) \subset$ $\operatorname{Ker}\left(i_{2 *}\right)$. Since $g_{*}$ is surjective, we must therefore have $g_{*} \operatorname{Ker}\left(i_{1 *}\right)=\operatorname{Ker}\left(i_{2 *}\right)$, so $\operatorname{dim}\left(\operatorname{Ker}\left(i_{1 *}\right)\right) \geq \operatorname{dim}\left(\operatorname{Ker}\left(i_{2 *}\right)\right)$, from which the lemma follows. 


\section{Reduction to a once-punctured torus}

We are given an automorphism of a torus with an arbitrary number, $k$, of cone points. We denote this orbifold $T\left(n_{1}, \ldots, n_{k}\right)$, where $n_{i}$ is the order of the $i$-th cone point. Let $\mathcal{M}\left(T\left(n_{1}, \ldots, n_{k}\right)\right)$ be the mapping class group of $T\left(n_{1}, \ldots, n_{k}\right)$. In general, these groups are rather complicated. However, the mapping class group of a torus with a single cone point is quite simple, being isomorphic to $S L_{2}(\mathbb{Z})$

Let $\mathcal{M}_{0}\left(T\left(n_{1}, \ldots, n_{k}\right)\right)$ denote the finite-index subgroup of the mapping class group which consists of those automorphisms which fix all the cone points of $T\left(n_{1}, \ldots, n_{k}\right)$. The following elementary fact allows us to pass to the simpler case of a single cone point.

Lemma 3.1 For any $i$, there is a homomorphism $\theta_{i}: \mathcal{M}_{0}\left(T\left(n_{1}, \ldots, n_{k}\right)\right)$ onto $\mathcal{M}\left(T\left(n_{i}\right)\right)$.

Proof Let $f \in \mathcal{M}_{0}\left(T\left(n_{1}, \ldots, n_{k}\right)\right)$. Since $f$ fixes the cone points, it restricts to a map on the punctured surface which is the complement of all the cone points except the $i$ th one. After filling in these punctures, there is an induced map $\theta_{i}(f)$ on $T_{n_{i}}$. It is easy to see that this is well-defined, surjective, and a homomorphism.

Lemma 3.2 Let $f \in \mathcal{M}_{0}\left(T\left(n_{1}, \ldots, n_{k}\right)\right)$. Then there is a surjective homomorphism from $\pi_{1} M_{f} \rightarrow \pi_{1} M_{\theta_{i} f}$.

Proof Let $F$ be the punctured surface obtained from $T\left(n_{1}, \ldots, n_{k}\right)$ by removing all the cone points. Let $x_{1}, \ldots, x_{k} \in \pi_{1} F$ be loops around the $k$ cone points, and complete these to a generating set with loops $x_{k+1}, x_{k+2}$. We have:

$$
\begin{aligned}
\pi_{1} M_{f} \cong<x_{1}, \ldots, x_{k+2}, t>/<t x_{1} t^{-1} & =f x_{1}, \ldots, t x_{k+2} t^{-1}=f x_{k+2}, \\
x_{1}^{n_{1}} & =\ldots=x_{k}^{n_{k}}=1>.
\end{aligned}
$$

From this presentation a presentation for $\pi_{1} M_{\theta_{i} f}$ may be obtained by adding the additional relations $x_{j}=i d$, for all $j \leq k, j \neq i$.

Corollary 3.3 Let $f \in \mathcal{M}\left(T\left(n_{1}, \ldots, n_{k}\right)\right)$. Then there is a finite index subgroup of $\pi_{1} M_{f}$ which maps onto $\pi_{1} M_{g}$, where $g$ is an automorphism of a torus with a single cone point.

Proof By passing to a finite-index subgroup, we may replace $f$ with a power, and then apply Lemma 3.2. 


\section{Increasing the first Betti number by at least one}

Before proving Theorem 1.2, we first prove:

Lemma 4.1 Let $M_{f}$ be as in the statement of Theorem 1.2. Then $v b_{1}\left(M_{f}\right)>b_{1}\left(M_{f}\right)$.

We remark that this result, combined with Lemma 2.3 and the arguments in the proof of Cor 1.4, implies that the first Betti number of a genus 2 bundle can be increased by at least 1 .

By Corollary 3.3, Lemma 4.1 will follow from the following lemma.

Lemma 4.2 Let $f \in \mathcal{M}(T(n))$ be an automorphism of a torus with a single cone point. Then $M_{f}$ has a finite cover $\widetilde{M_{f}}$ such that $b_{1}\left(\widetilde{M_{f}}\right)>b_{1}\left(M_{f}\right)$.

Proof of Lemma 4.2 We shall use $T$ to denote the once-punctured torus obtained by removing the cone point of $T(n)$. There is an induced map $f: T \rightarrow$ $T$. In order to construct covers of $T$, we require the techniques of [6]. For convenience, the relevant ideas and notations are contained in the appendix. In what follows, we assume familiarity with this material.

Case $1 n=2$

We let $J$ denote the subgroup of the mapping class group of $T$ generated by $D_{x}$ and $D_{y}^{4}$. By Lemma 8.2, $J$ has finite index, so we may assume, after replacing $f$ with a power, that the map $f: T \rightarrow T$ lies in $J$.

As explained in the appendix, any four permutations $\sigma_{1}, \ldots, \sigma_{4}$ on $r$ letters will determine a $4 r$-fold cover $\widetilde{T}$ of $T$. We set:

I $\sigma_{2}=\sigma_{1}^{-1}$ and $\sigma_{4}=\sigma_{3}^{-1}$,

so $f$ lifts to $\widetilde{T}$ by Lemma 8.3 . We shall require every lift of $\partial T$ to unwrap once or twice in $\widetilde{T}$. This property is equivalent to the following:

II $\left(\sigma_{i} \sigma_{i+1}^{-1}\right)^{2}=i d$ for all $i$.

To find permutations satisfying I and II, we consider the abstract group generated by the symbols $\sigma_{1}, \ldots, \sigma_{4}$, satisfying relations I and II. If this group surjects a finite group $G$, then we may take the associated permutation representation, and obtain permutations $\sigma_{1}, \ldots, \sigma_{4}$ on $|G|$ letters satisfying I and II. In the case under consideration, we may take $G$ to be a cyclic group of order 4 . This leads 


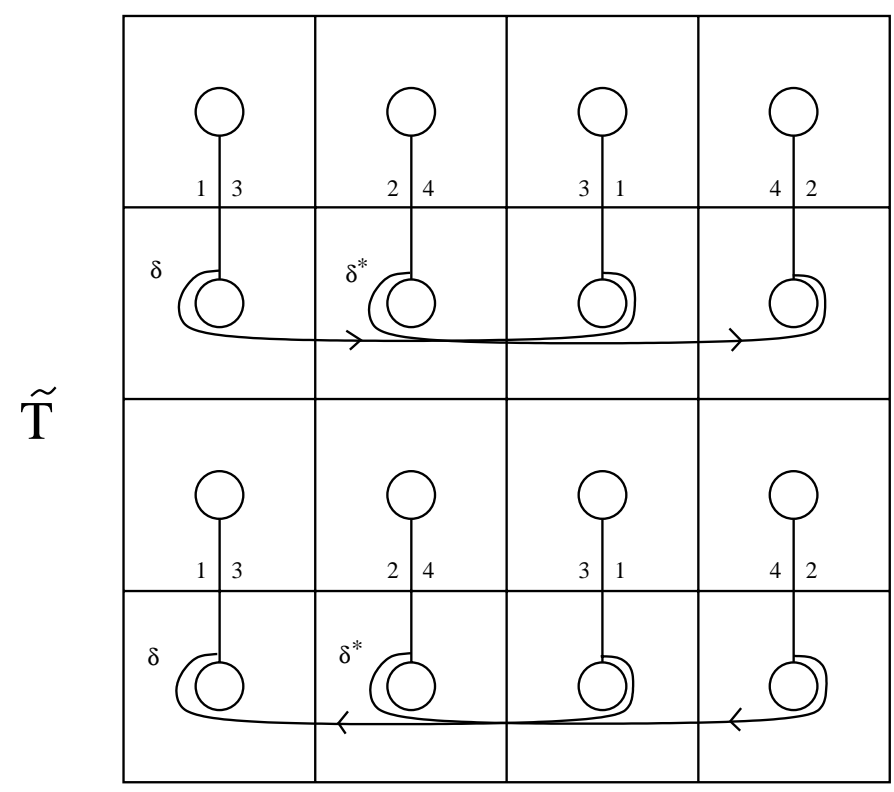

$\downarrow$

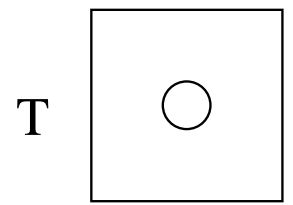

Figure 2: The cover $\widetilde{T}$ of $T$

to the representation $\sigma_{1}=\sigma_{3}=(1234), \sigma_{2}=\sigma_{4}=\sigma_{1}^{-1}$. The associated cover is pictured in Figure 2.

Lemma 8.3 now guarantees that non-trivial fixed classes in $H_{1}(\widetilde{T}, \partial \widetilde{T})$ exist. Rather than invoke the lemma, however, we shall give the explicit construction for this simple case. Consider the classes $[\delta],\left[\delta^{*}\right] \in H_{1}(\widetilde{T}, \partial \widetilde{T})$ pictured in Figure 2 .

Proposition $4.3[\delta],\left[\delta^{*}\right] \in H_{1}(\widetilde{T}, \partial \widetilde{T})$ are non-zero classes which are fixed by any element of $J$.

In the proof, the notation $I(.,$.$) stands for the algebraic intersection pairing on$ 
$H_{1}$ of a surface.

Proof The fact that $[\delta]$ and $\left[\delta^{*}\right]$ are non-peripheral follows from the fact that $I\left([\delta],\left[\delta^{*}\right]\right)=2$. The loops $\delta$ and $\delta^{*}$ have algebraic intersection number 0 with each lift of $y$, and therefore their homology classes are fixed by the lift of $D_{y}^{4}$.

By Property I and by Lemma $8.1, D_{x}$ lifts to $\widetilde{T}$, and acts as the identity on Rows 2 and 4 . In particular, $[\delta]$ and $\left[\delta^{*}\right]$ are fixed by the lift of $D_{x}$. Therefore, $[\delta]$ and $\left[\delta^{*}\right]$ are fixed by any element of $J$.

Since $\partial T$ unwraps exactly twice in every lift to $\widetilde{T}$, then by filling in the punctures of $\widetilde{T}$, we obtain a manifold cover $\widetilde{T(n)}$ of $T(n)$. Since $f$ lifts to $\widetilde{T}$, then $f$ lifts to $\widetilde{T(n)}$. An application of Propositions 4.3 and 2.1 finishes the proof of Lemma 4.2 in this case.

Case $2 \quad n \geq 3$

In this case, we shall require a cover of $T$ in which the boundary components unwrap $n$ times. We construct a cover $\widetilde{T}$ of $T$, mimicking the construction given in Case 1 . We start with the standard $\mathbb{Z} / r \times \mathbb{Z} / 4$ cover of $T$, and alter it by cutting and pasting in a manner specified by permutations $\sigma_{1}, \ldots, \sigma_{4}$. By raising $f$ to a power, we may assume that $f$ lies in $J$, the subgroup of the mapping class group of $T$ generated by $D_{x}$ and $D_{y}^{4}$. Again, $D_{y}^{4}$ lifts to Dehn twists in the lifts of $y$. Lemma 8.1 shows that, if we set:

I $\sigma_{2}=\sigma_{1}^{-1}$ and $\sigma_{4}=\sigma_{3}^{-1}$,

then $D_{x}$ lifts also, so $f$ lifts. To ensure that the boundary components unwrap appropriately, we also require $\left(\sigma_{i} \sigma_{i+1}^{-1}\right)^{n}=1$. Combining this with condition I gives:

II $\left(\sigma_{1}\right)^{2 n}=\left(\sigma_{3}\right)^{2 n}=\left(\sigma_{1} \sigma_{3}\right)^{n}=1$.

If we consider the symbols $\sigma_{1}$ and $\sigma_{3}$ as representing abstract group elements, Conditions I and II determine a hyperbolic triangle group $\Gamma$. It is a well-known property of triangle groups that $\sigma_{1}, \sigma_{3}$, and $\sigma_{1} \sigma_{3}$ will have orders in $\Gamma$ as given by the relators in Condition I. Also, it is a standard fact that in this case $\Gamma$ is infinite, and residually finite. Therefore, $\Gamma$ surjects arbitrarily large finite groups such that the images of $\sigma_{1}, \sigma_{3}$, and $\sigma_{1} \sigma_{3}$ have orders $2 n, 2 n$, and $n$, respectively. Let $G$ be such a finite quotient, of order $N$ for some large number $N$. By taking the left regular permutation representation of $G$, we obtain permutations on $N$ letters satisfying Conditions I and II, as required. 
Let $V$ denote the subspace of $H_{1}(\widetilde{T}, \partial \widetilde{T})$ fixed by $f$. By Lemma $8.3, \operatorname{dim}(V) \geq$ 2genus $\left(R_{2}\right)$, where $R_{2}$ is the subsurface of $\widetilde{T}$ corresponding to Row 2 .

The formula for genus is:

$$
\operatorname{genus}\left(R_{2}\right)=\frac{1}{2}\left(2-\chi\left(R_{2}\right)-\left(\# \text { of punctures of } R_{2}\right)\right) \text {. }
$$

Any permutation $\sigma$ decomposes uniquely as a product of disjoint cycles; we denote the set of these cycles by $\operatorname{cycles}(\sigma)$. The punctures of $R_{2}$ are in 11 correspondence with the cycles of $\sigma_{1}, \sigma_{3}$ and $\sigma_{3} \sigma_{1}$. Also, since $R_{2}$ is an $N$-fold cover of a thrice-punctured sphere, we have the Euler characteristic $\chi\left(R_{2}\right)=-N$, and so we get:

$$
\operatorname{genus}\left(R_{2}\right)=\frac{1}{2}\left(2+N-\left(\left|\operatorname{cycles}\left(\sigma_{1}\right)\right|+\left|\operatorname{cycles}\left(\sigma_{3}\right)\right|+\left|\operatorname{cycles}\left(\sigma_{1} \sigma_{3}\right)\right|\right)\right) .
$$

Recall that an $m$-cycle is a permutation which is conjugate to (1...m). Any permutation $\sigma$ coming from the left regular permutation representation of $G$ decomposes as a product of $N / \operatorname{order}(\sigma)$ disjoint $\operatorname{order}(\sigma)$-cycles, and therefore

$$
\left|\operatorname{cycles}\left(\sigma_{1} \sigma_{3}\right)\right|=2\left|\operatorname{cycles}\left(\sigma_{1}\right)\right|=2\left|\operatorname{cycles}\left(\sigma_{3}\right)\right|=|G| / n=N / n \text {. }
$$

Combining the above formulas gives

$$
\operatorname{dim}(V) \geq 2+N(1-2 / n) .
$$

So $\operatorname{dim}(V)$ can be made arbitrarily large.

There are corresponding covers $\widetilde{T}$ of $T$, and $\widetilde{T(n)}$ of $T(n)$. Proposition 2.1 then shows that, in this case, $v b_{1}\left(M_{f}\right)=\infty$.

\section{$5 \quad$ Infinite virtual first Betti number}

In this section we prove Theorem 1.2.

Lemma 5.1 Let $f \in \mathcal{M}(T(n))$ be an automorphism of a torus with a single cone point. Then $v b_{1}\left(M_{f}\right)=\infty$.

Proof In the course of proving Lemma 4.1, we actually proved Lemma 5.1 in the case $n>2$, so we assume $n=2$. The proof of Lemma 4.1 also shows how to increase $b_{1}\left(M_{f}\right)$ by at least 2 ; next we will show how to increase $b_{1}\left(M_{f}\right)$ by at least 4 , and fianlly we will indicate how to iterate this process to increase $b_{1}\left(M_{f}\right)$ arbitrarily.

Again, let $T$ be the once punctured torus obtained by removing the cone point of $T(2)$. By replacing $T$ with a 2 -fold cover, and by replacing $f$ with a power 
(to make it lift), we may assume that $T$ has two boundary components, denoted $\alpha_{1}, \alpha_{2}$. By again replacing $f$ with a power, we may assume that $f$ fixes both $\alpha_{i}$ 's.

Let $T_{1}^{+}$denote the once punctured torus obtained by filling in $\alpha_{2}$. Since $f$ fixes both $\alpha_{i}$ 's, there is an induced automorphism $f: T_{1}^{+} \rightarrow T_{1}^{+}$. Let $\widetilde{T_{1}^{+}}$be the 16-fold cover of $T_{1}^{+}$as constructed in the previous section, and let $\delta_{1}^{+}, \delta_{1}^{+*}$ be the loops constructed previously, whose homology classes are fixed by (a power of) $f$.

Let $\widetilde{T}_{1}$ denote the cover of $T$ corresponding to $\widetilde{T_{1}^{+}}$(see Figure 3 ). By replacing $f$ with a power, we may assume that $f$ lifts to $\widetilde{T}_{1}$. Let $\delta_{1}, \delta_{1}^{*} \subset \widetilde{T}_{1}$ denote the pre-images of $\delta_{1}^{+}$and $\delta_{1}^{+*}$ under the natural inclusion map (after an isotopy, we may assume that $\delta_{1}^{+}$and $\delta_{1}^{+*}$ are disjoint from all filled-in punctures, so that $\delta_{1}$ and $\delta_{1}^{*}$ are in fact loops).

Since $\left[\delta_{1}^{+}\right]$and $\left[\delta_{1}^{+*}\right]$ are fixed classes in $H_{1}\left(\widetilde{T}_{1}^{+}, \partial \widetilde{T}_{1}^{+}\right)$, then by Proposition 2.2, $\left[\delta_{1}\right]$ and $\left[\delta_{1}^{*}\right]$ are fixed classes in $H_{1}\left(\widetilde{T}_{1}, \partial \widetilde{T}_{1}\right)$. Note that $I\left(\left[\delta_{1}\right],\left[\delta_{1}^{*}\right]\right)=2$.

Starting with $\alpha_{2}$ instead of $\alpha_{1}$, we may perform the analogous construction to obtain a cover $\widetilde{T}_{2}$ of $T$ containing fixed classes $\left[\delta_{2}\right],\left[\delta_{2}^{*}\right] \in H_{1}\left(\widetilde{T}_{2}, \partial \widetilde{T}_{2}\right)$, with algebraic intersection number 2. Moreover, as indicated by Figure $3, \delta_{2}$ and $\delta_{2}^{*}$ may be chosen so that their projections to $T$ are disjoint from the projections of $\delta_{1}$ and $\delta_{1}^{*}$ to $T$.

Let $\widetilde{T}$ denote the cover of $T$ with covering group $\pi_{1}\left(\widetilde{T_{1}}\right) \cap \pi_{1}\left(\widetilde{T_{2}}\right)$. Since $f$ lifts to $\widetilde{T}_{1}$ and $\widetilde{T}_{2}$, then $f$ also lifts to $\widetilde{T}$. Let $\widetilde{\delta}_{i}$ and $\widetilde{\delta}_{i}^{*}$ denote the full pre-images in $\widetilde{T}$ of $\delta_{i}$ and $\delta_{i}^{*}$, respectively. Recall that by construction, $\delta_{i}$ and $\delta_{i}^{*}$ have the following properties, for $i=1,2$ :

(1) $\left[\delta_{i}\right],\left[\delta_{i}^{*}\right] \in H_{1}\left(\widetilde{T}_{i}, \partial \widetilde{T}_{i}\right)$ are fixed classes.

(2) $I\left(\left[\delta_{i}\right],\left[\delta_{i}^{*}\right]\right) \neq 0$.

(3) The projections of $\delta_{1} \cup \delta_{1}^{*}$ and $\delta_{2} \cup \delta_{2}^{*}$ to $T$ are disjoint.

Therefore, by elementary covering space arguments, we deduce that $\widetilde{\delta}_{i}$ and $\widetilde{\delta}_{i}^{*}$ have the following properties for $i=1,2$ :

(1) $\left[\widetilde{\delta}_{i}\right],\left[\widetilde{\delta}_{i}^{*}\right] \in H_{1}(\widetilde{T}, \partial \widetilde{T})$ are fixed classes.

(2) $I\left(\left[\widetilde{\delta}_{i}\right],\left[\widetilde{\delta}_{i}^{*}\right]\right) \neq 0$. 

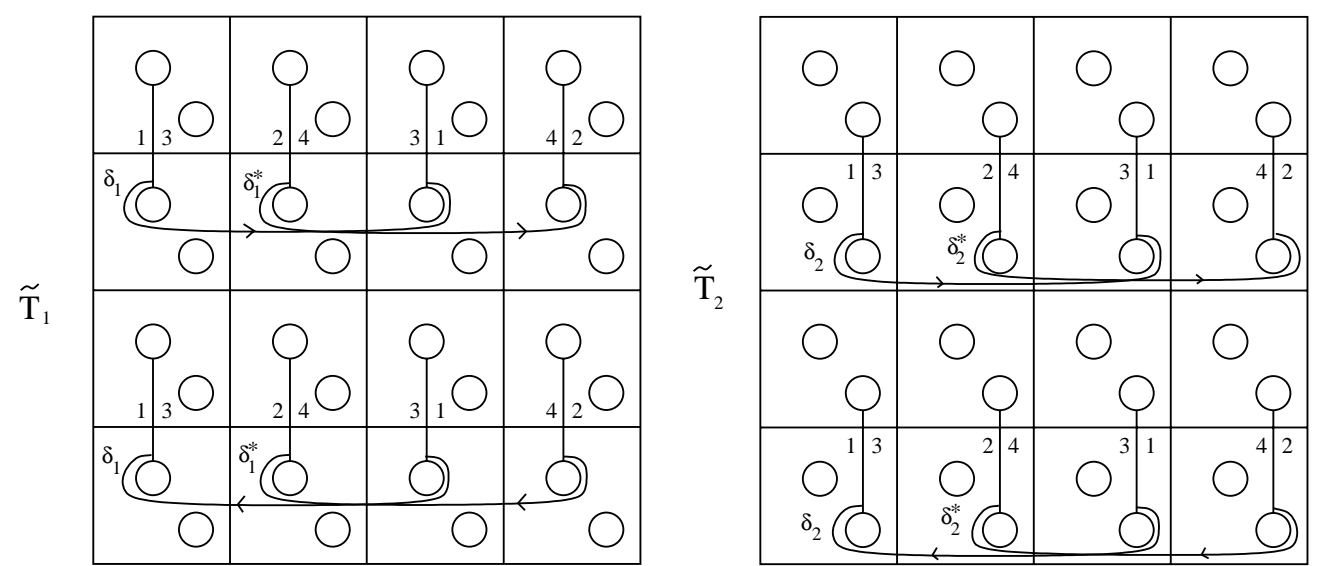

$\downarrow$

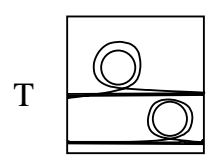

Figure 3: We may arrange for $\delta_{1} \cup \delta_{1}^{*}$ and $\delta_{2} \cup \delta_{2}^{*}$ to have disjoint projections.

(3) $\widetilde{\delta}_{1} \cup \widetilde{\delta}_{1}^{*}$ and $\widetilde{\delta}_{2} \cup \widetilde{\delta}_{2}^{*}$ are disjoint.

Claim The subspace of $H_{1}(\widetilde{T}, \partial \widetilde{T})$ on which $f$ acts trivially has dimension at least 4.

Proof By Property (1) above, it is enough to show that the vectors $\left[\delta_{1}\right],\left[\delta_{1}^{*}\right]$, $\left[\delta_{2}\right],\left[\delta_{2}^{*}\right]$ are linearly independent in $H_{1}(\widetilde{T}, \partial \widetilde{T})$. Let $V_{i}$ be the space generated by $\left[\delta_{i}\right]$ and $\left[\delta_{i}^{*}\right]$. It follows from Property $(2)$ that $\operatorname{dim}\left(V_{i}\right)=2$. By Property (3), we have $I\left(v_{1}, v_{2}\right)=0$ for any $v_{1} \in V_{1}$ and $v_{2} \in V_{2}$. The intersection form $I$ restricted to $V_{2}$ is a non-zero multiple of the form $\left(\begin{array}{ll}0 & 1 \\ -1 & 0\end{array}\right)$, which is nonsingular. So, for any $v_{2} \in V_{2}$, there is an element $v_{2}^{*} \in V_{2}$ such that $I\left(v_{2}, v_{2}^{*}\right) \neq$ 0 . Therefore $V_{1} \cap V_{2}=\emptyset$, so the four vectors are linearly independent, and the claim follows.

Each lift of the puncture $\alpha_{1}$ unwraps twice in $\widetilde{T_{1}}$ and once in $\widetilde{T_{2}}$. Therefore each lift of $\alpha_{1}$ unwraps twice in $\widetilde{T}$; similarly, each lift of $\alpha_{2}$ unwraps twice in 
$\widetilde{T}$. Hence there is an induced manifold cover $\widetilde{T(2)}$ of $T(2)$ obtained by filling in the punctures of $\widetilde{T}$. There is then an induced manifold cover $\widetilde{M_{f}}$ of $M_{f}$, and by Proposition 2.1, $b_{1}\left(\widetilde{M_{f}}\right) \geq 4+1$.

The proof of the general result is similar. We start with an arbitrary positive integer $k$, and replace $T$ with a $k$-times punctured torus.

We then obtain, for each $i \leq k$, a cover $\widetilde{T}_{i}$ of $T$, such that each puncture of $T$ unwraps once or twice in $\widetilde{T}_{i}$. We construct fixed classes $\left[\delta_{i}\right],\left[\delta_{i}^{*}\right] \in H_{1}\left(\widetilde{T}_{i}, \partial \widetilde{T}_{i}\right)$ with algebraic intersection number 2 , so that the projection of $\delta_{i} \cup \delta_{i}^{*}$ to $T$ is disjoint from the projection of $\delta_{j} \cup \delta_{j}^{*}$ whenever $i \neq j$ (see Figure 4).

By an argument similar to the one given in the $k=2$ case, we conclude that there is a $2 k$-dimensional space in $H_{1}(\widetilde{T}, \partial \widetilde{T})$ on which $f$ acts trivially. Since every puncture of $T$ unwraps twice in $\widetilde{T}$, there is an induced manifold cover $\widetilde{T(2)}$ of $T(2)$ obtained by filling in the punctures of $\widetilde{T}$. Therefore there is an induced bundle cover of $M_{f}$, and by Proposition $2.1, b_{1}\left(\widetilde{M_{f}}\right) \geq 2 k+1$. Since $k$ is an arbitrary positive integer, the result follows.

Proof of Theorem 1.2 This is an application of Lemma 5.1 and Corollary 3.3

\section{Proof of Theorem 1.5}

We sketch the proof that $M$ has a finite cover $\widetilde{M}$ with $b_{1}(\widetilde{M})>b_{1}(M)$. The generalization to $v b_{1}(M)=\infty$ then follows by direct analogy with the proof of Theorem 1.2. Recall the construction of the cover $\widetilde{F}$ of $F$ in the case of a hyper-elliptic monodromy: we remove a neighborhood of the fixed points of $\tau$ to obtain a punctured surface $F^{-}$. The surface $F^{-}$double covers a planar surface $P$; we construct a punctured torus $T$ which double covers $P$, and then a 16 -fold cover $\widetilde{T}$ of $T$. The cover $\widetilde{F}$ of $F$ corresponds to $\pi_{1} \widetilde{T} \cap \pi_{1} F^{-}$. A loop $\delta \subset \widetilde{T}$ is constructed, whose full pre-image $\widetilde{\delta}$ in $\widetilde{F}$ represents a homology class which is fixed by (a power of) any element of $H=<D_{x_{1}}, \ldots, D_{x_{2 g}}>$.

The covers $T$ and $\widetilde{T}$ of $P$ are not characteristic. Any element $h$ of $H$ sends $T$ to a cover $h T$ of $P$, and $\widetilde{T}$ to a cover $h \widetilde{T}$ of $h T$; let $h_{0}=i d, h_{1}, \ldots, h_{n} \in H$ denote the elements necessary for a full orbit of $\widetilde{T}$. Let $K_{j} \subset H_{1}\left(h_{j} \widetilde{T}, \partial h_{j} \widetilde{T}\right)$ denote the kernel of the projection to $H_{1}\left(h_{j} T, \partial h_{j} T\right)$. By construction, we have $\delta \in K_{0}$. Let $\gamma$ be the loop pictured in Figure 1, and let $p \gamma$ denote the projection of $\gamma$ to $P$. We claim that every component of the pre-image of $p \gamma$ 

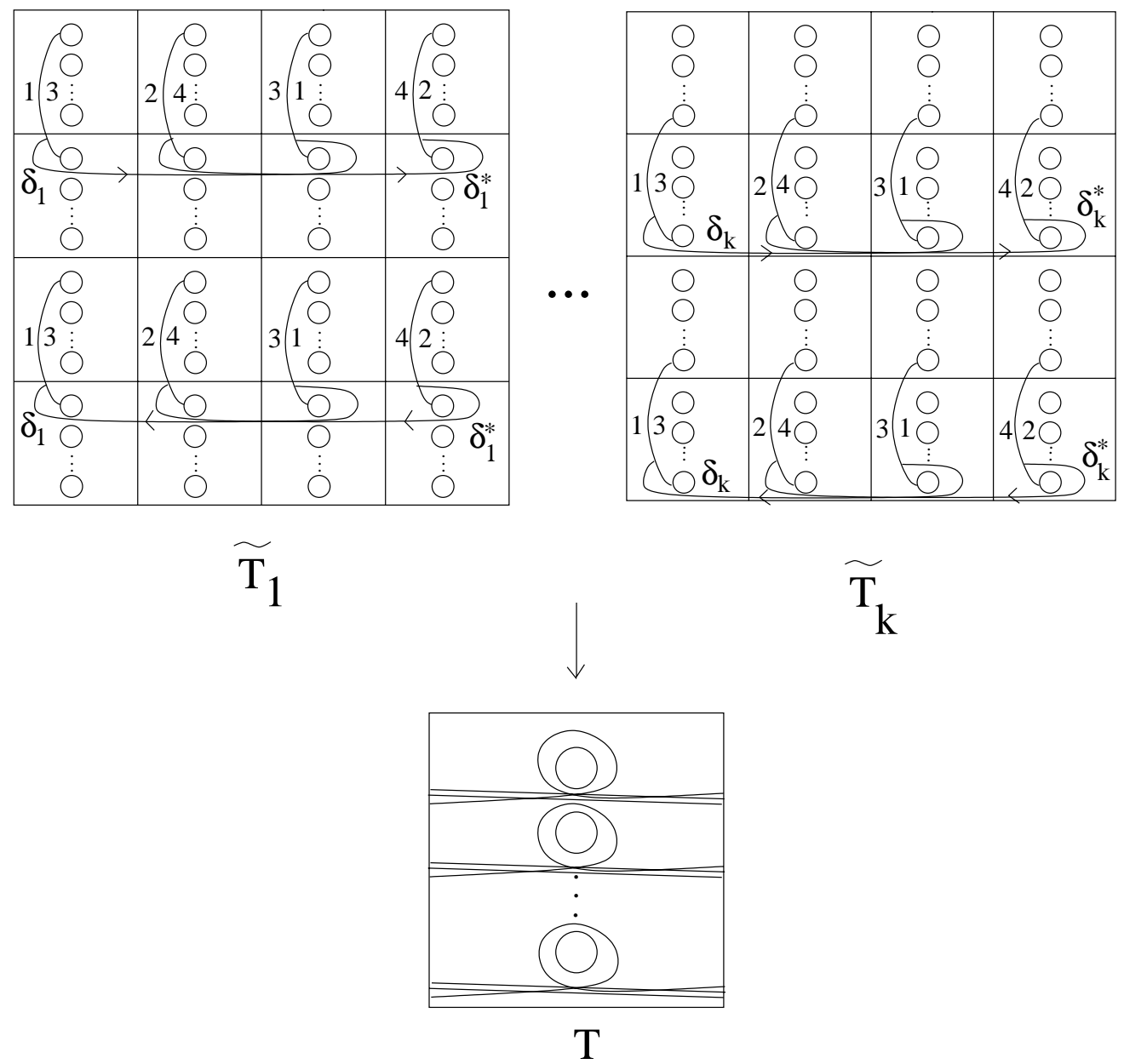

Figure 4: Each boundary component of $T$ gives rise to a different cover.

in $h_{j} \widetilde{T}$ has intersection number 0 with every class in $K_{j}$ : this may be checked by constructing an explicit basis for the $K_{j}$ 's.

Now, fix an element $h \in H$. The $K_{j}$ 's are permuted by $H$, so $h[\delta]$ has 0 intersection number with each component of the pre-image of $p \gamma$ in $h \widetilde{T}$. Therefore, every component of the pre-image of $\gamma$ in $h \widetilde{F}$ has 0 intersection number with $h[\widetilde{\delta}]$. Since the pre-images of $\gamma$ unwrap at most 8 times, we see that $D_{\gamma}^{8}$ lifts to Dehn twists in $h \widetilde{F}$, and fixes $h[\widetilde{\delta}]$. Therefore, the action of $f$ on $[\widetilde{\delta}]$ is unchanged if we remove all the $D_{\gamma}^{8}$ 's, and we deduce from the hyper-elliptic case that, for some integer $m, f^{m}$ lifts to a map $\widetilde{f^{m}}$ such that $\widetilde{f^{m}}{ }_{*}[\widetilde{\delta}]=[\widetilde{\delta}]$. 


\section{Proof of Theorem 1.7}

Let $K$ be the knot $9_{32}$ in Rolfsen's tables, and let $M=S^{3}-K$. The computer program SnapPea shows that $M$ has no symmetries. A knot complement is said to have hidden symmetries if it is an irregular cover of some orbifold. In our example, $M$ has no hidden symmetries, since by [7], a hyperbolic knot complement with hidden symmetries must have cusp parameter in $\mathbb{Q}(\sqrt{-1})$ or $\mathbb{Q}(\sqrt{-3})$, but it is shown in [11] that the cusp field of $M$ has degree 29 .

Since $M$ has no symmetries or hidden symmetries, and is non-arithmetic (see [9]), it follows from results of Margulis that $M$ is the unique minimal orbifold in its commensurability class.

Let $M(0, n)$ be the orbifold filling on $K$ obtained by setting the $n$-th power of the longitude to the identity. Then, by Corollary 3.3 of [10], if $n$ is large enough, $M(0, n)$ is a hyperbolic orbifold which is minimal in its commensurability class. We choose a large $n$ which satisfies this condition and is odd.

Since $K$ has monic Alexander polynomial and fewer than 11 crossings, it is fibered (see [5]), and therefore $M(0, n)$ is 2 -orbifold bundle over $S^{1}$. This orbifold bundle is finitely covered by a manifold which fibers over $S^{1}$; let $f: F \rightarrow$ $F$ denote the monodromy of this fibration. We claim that no power of $f$ lifts to become hyper-elliptic in any cover of $F$.

For suppose such a cover $\widetilde{F}$ of $F$ exists. Then there is an associated cover $\widetilde{M(0, n)}$ of $M(0, n)$, and an involution $\tau$ on $\widetilde{M(0, n)}$ with one-dimensional fixed point set. The quotient $Q=\widetilde{M(0, n)} / \tau$ is an orbifold whose singular set is a link labeled 2, which is commensurable with $M(0, n)$. By minimality, $Q$ must cover $M(0, n)$. But this is impossible, since every torsion element of $M(0, n)$ has odd order, by our choice of $n$.

\section{Appendix: Constructing Covers of Punctured Tori}

We review here the relevant material from [6]. This builds on work of Baker $([1],[2])$.

We are given a punctured torus $T$ and a monodromy $f$, and we wish to find finite covers of $T$ to which $f$ lifts. Let $x$ and $y$ be the generators for $\pi_{1} T$ pictured in Figure 5. Let $r$ and $s$ be positive integers, and let $\hat{T}$ be the $r s-$ fold cover of $T$ associated to the kernel of the map $\phi: \pi_{1}(T) \rightarrow \mathbb{Z}_{r} \times \mathbb{Z}_{s}$, with $\phi([x])=(1,0)$ and $\phi([y])=(0,1)$ (see Figure 5). 


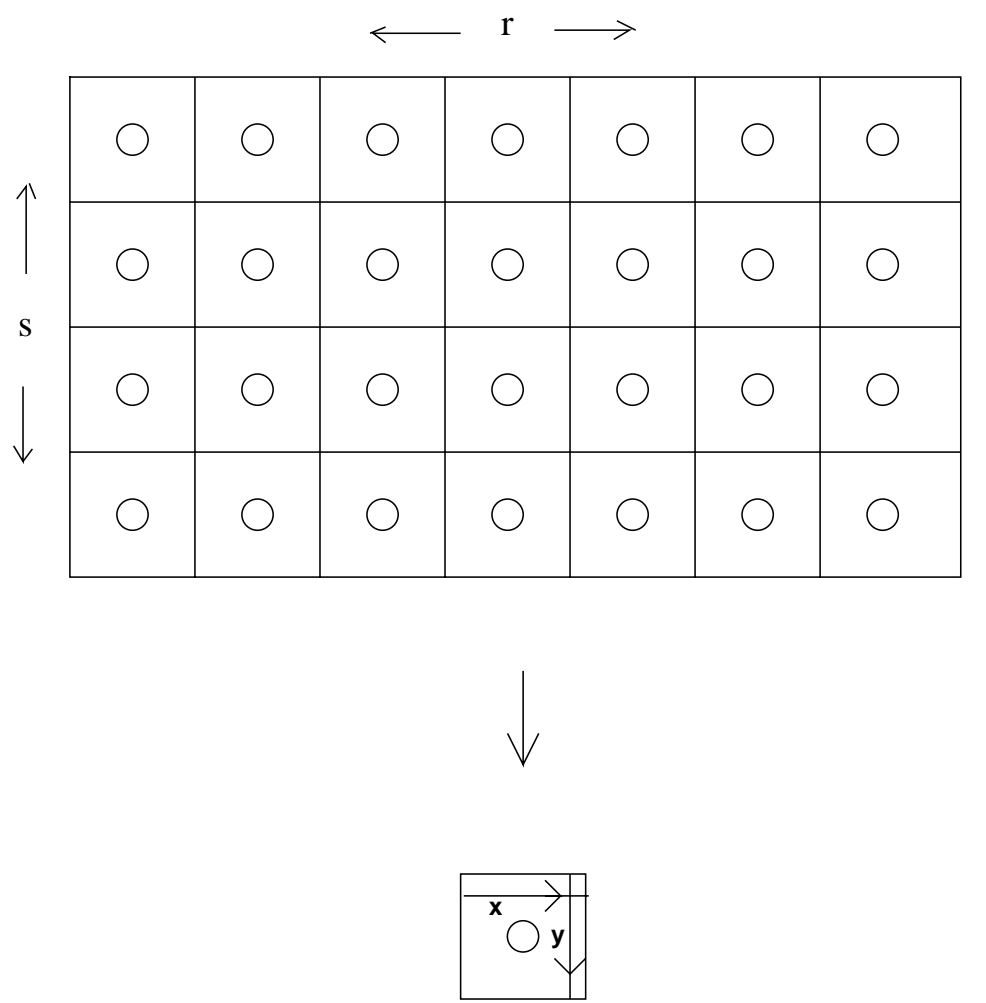

Figure 5: The cover $\hat{T}$ of $T$

Now we create a new cover, $\widetilde{T}$, of $T$ by making vertical cuts in each row of $\hat{T}$, and gluing the left side of each cut to the right side of another cut in the same row. An example is pictured in Figure 6, where the numbers in each row indicate how the edges are glued.

We now introduce some notation to describe the cuts of $\widetilde{T}$ (see Figure 6 ). $\widetilde{T}$ is naturally divided into rows, which we label $1, \ldots, s$. The cuts divide each row into pieces, each of which is a square minus two half-disks; we number them $1, \ldots, r$. If we slide each point in the top half of the $i^{\text {th }}$ row through the cut to its right, we induce a permutation on the pieces $\{1, \ldots, r\}$, which we denote $\sigma_{i}$. Thus the cuts on $\widetilde{T}$ may be encoded by elements $\sigma_{1}, \ldots, \sigma_{s} \in S_{r}$, the permutation group on $r$ letters.

Let $D_{x}$ and $D_{y}$ be the right-handed Dehn twists in $x$ and $y$, which generate the mapping class group of $T$. We observe that, regardless of the choice of $\sigma_{i}$ 's, $D_{y}^{s}$ lifts to a product of Dehn twists in $\widetilde{T}$. It will be useful to have a condition 


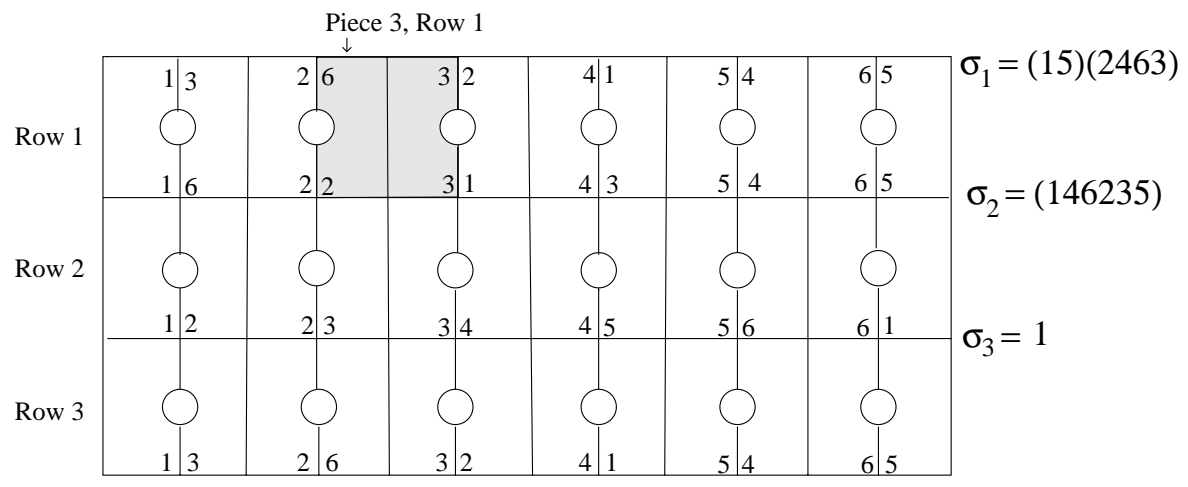

Figure 6: The permutations encode the combinatorics of the gluing

on the $\sigma_{i}$ 's which will guarantee that $D_{x}$ lifts to $\widetilde{T}$. The following lemma (in slightly different form) appears in [6].

Lemma 8.1 $D_{x}$ lifts to $\widetilde{D}_{x}: \widetilde{T} \rightarrow \widetilde{T}$ if

(1) $\sigma_{1} \ldots \sigma_{i}$ commutes with $\sigma_{i+1}$ for $i=1, \ldots, s-1$, and

(2) $\sigma_{1} \ldots \sigma_{s}=1$.

Moreover, if these conditions are satisfied, then we may choose $\widetilde{D}_{x}$ so that its action on the interior of the $i$ th row of $\widetilde{T}$ corresponds to the permutation $\sigma_{1} \ldots \sigma_{i}$.

Proof We shall attempt to lift $D_{x}$ explicitly to a sequence of "fractional Dehn twists" along the rows of $\widetilde{T}$. Let $\widetilde{x}_{i}$ denote the disjoint union of the lifts of $x$ to the $i^{\text {th }}$ row of $\widetilde{T}$. We first attempt to lift $D_{x}$ to row 1 , twisting one slot to the right along $\widetilde{x}_{1}$. Considering the effect of this action on the bottom half of row 1, we find the cuts there are now matched up according to the permutation $\sigma_{1}^{-1} \sigma_{2} \sigma_{1}$. Thus, for $D_{x}$ to lift to row 1 we assume $\sigma_{1}$ and $\sigma_{2}$ commute. We now twist along $\widetilde{x}_{2}$. The top halves of the squares in row 2 are moved according to the permutation $\sigma_{1} \sigma_{2}$, and the lift will extend to all of row 2 if $\sigma_{3}$ commutes with $\sigma_{1} \sigma_{2}$. We continue in this manner, obtaining the conditions in 1 . After we twist through $\widetilde{x}_{n}$, we need to be back where we started in row 1 ; if the permutations satisfy the additional condition $\sigma_{1} \sigma_{2} \ldots \sigma_{s}=1$, then this is the case, and we have succeeded in lifting $D_{x}$. Note that in the course of constructing the lift, we have also verified the last assertion of the lemma.

For the purposes of this paper, we restrict attention to the case $s=4$. Consider the subgroup $J=<D_{x}, D_{y}^{4}>$ of the mapping class group of $T$. If $\sigma_{1}, \ldots, \sigma_{4}$ 
satisfy the conditions of Lemma 8.1, then any element of $J$ lifts to $\widetilde{T}$. What makes this useful is the following lemma.

Lemma 8.2 The subgroup $J$ has finite index in the mapping class group of $T$.

Proof The mapping class group of $T$ may be indentified with $S L_{2}(\mathbb{Z})$, and under this indentification, $J$ is the group generated by $\left(\begin{array}{ll}1 & 0 \\ 4 & 1\end{array}\right)$ and $\left(\begin{array}{ll}1 & 1 \\ 0 & 1\end{array}\right)$. Let $\gamma=\left(\begin{array}{ll}\sqrt{2} & 0 \\ 0 & \frac{1}{\sqrt{2}}\end{array}\right)$. Then $\gamma$ conjugates the generators of $J$ to $\left(\begin{array}{ll}1 & 0 \\ 2 & 1\end{array}\right)$ and $\left(\begin{array}{ll}1 & 2 \\ 0 & 1\end{array}\right)$, which are well known to generate the kernel of the reduction map from $S L_{2}(\mathbb{Z})$ to $S L_{2}(\mathbb{Z} / 2)$. Therefore $J$ is a finite co-area lattice in $S L_{2}(\mathbb{R})$, and therefore it has finite index in $S L_{2}(\mathbb{Z})$.

The next lemma shows that with some additional hypotheses on the $\sigma_{i}$ 's we are also guaranteed that the lifts of elements of $J$ fix non-peripheral homology classes of $\widetilde{T}$.

Lemma 8.3 Let $\widetilde{T}$ be as constructed above, and suppose $\sigma_{2}=\sigma_{1}^{-1}$ and $\sigma_{4}=\sigma_{3}^{-1}$. Let $f$ be an element of $J$. Then

(i) $f$ lifts to an automorphism $\widetilde{f}: \widetilde{T} \rightarrow \widetilde{T}$, and

(ii) For every non-peripheral loop $\ell$ in Row 2, there is a loop $\ell^{*}$ in Row 4, such that $\widetilde{f}_{*}\left[\ell \cup \ell^{*}\right]=\left[\ell \cup \ell^{*}\right] \neq[0] \in H_{1}(\widetilde{T}, \partial \widetilde{T})$.

Proof Assertion (i) is an immediate consequence of Lemma 8.1. To prove Assertion (ii), we explicitly construct the loop $\ell^{*}$, so that it intersects the same components of $\widetilde{y}$ as $\ell$ does, but with opposite orientations. Figure 7 indicates the procedure for doing this.

Therefore $\left[\ell \cup \ell^{*}\right]$ has 0 intersection number with each component of $\widetilde{y}$, and so it is fixed homologically by $\widetilde{D}_{y}^{4}$. Moreover, $\ell \cup \ell^{*}$ is entirely contained in Rows 2 and 4 , and Lemma 8.1 implies that the action of $\widetilde{D}_{x}$ is trivial there, so $\left[\ell \cup \ell^{*}\right]$ is also fixed by $\widetilde{D}_{x}$, and by every element of $J$. 

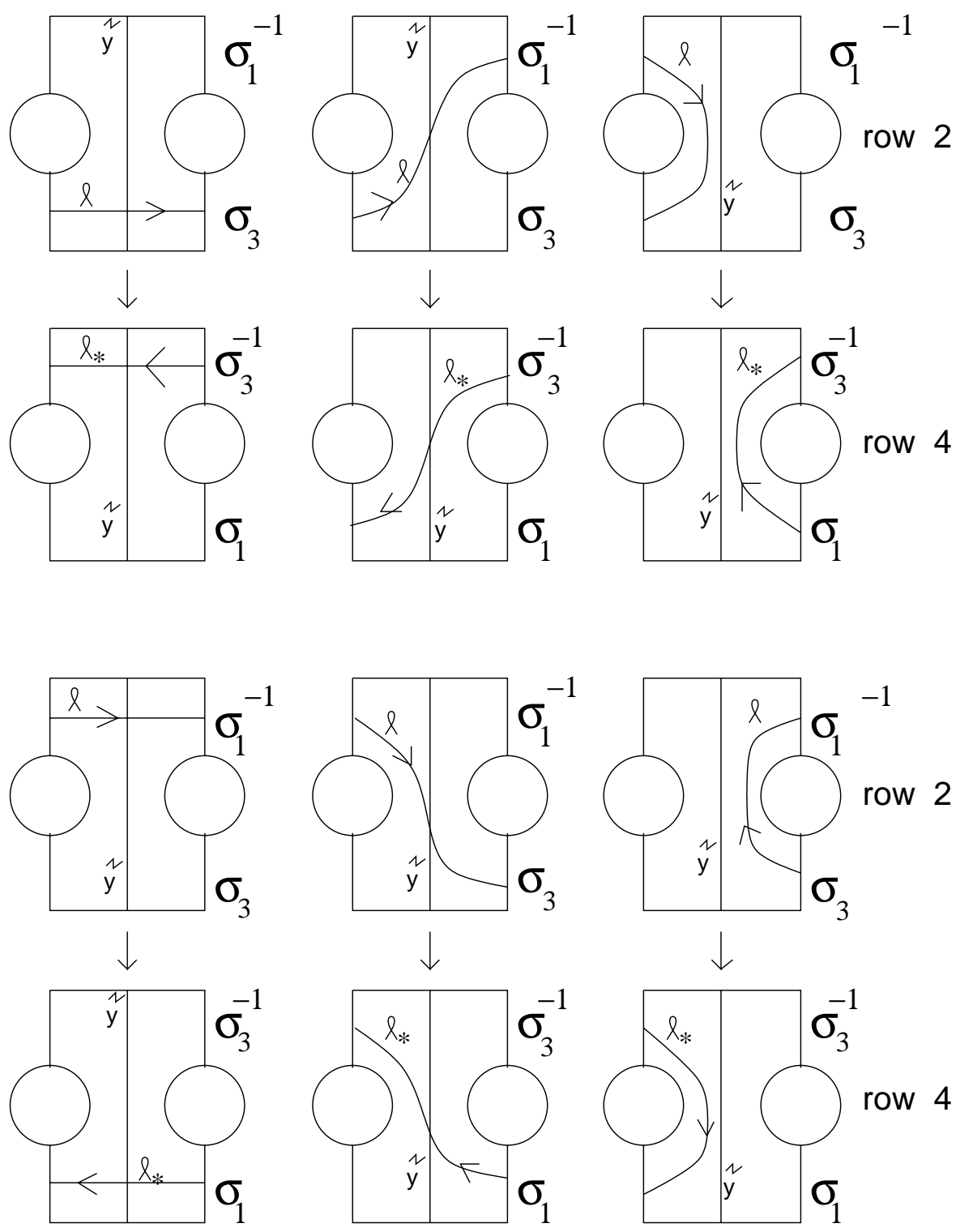

Figure 7: Corresponding to each segment of $\ell$, we construct a corresponding segment of $\ell_{*}$.

Geometry $8 \mathcal{T}$ Topology, Volume 6 (2002) 


\section{References}

[1] M Baker, Covers of Dehn fillings on once-punctured torus bundles, Proc. Amer. Math. Soc. 105 (1989) 747-754

[2] M Baker, Covers of Dehn fillings on once-punctured torus bundles II, Proc. Amer. Math. Soc. 110 (1990) 1099-1108

[3] D Gabai, On 3-manifolds finitely covered by surface bundles, from: "Lowdimensional Topology and Kleinian Groups (Coventry/Durham, 1984)", LMS Lecture Note Series 112, Cambridge University Press (1986)

[4] S P Humphries, Generators for the mapping class group, from: "Topology of Low-Dimensional Manifolds", Proceedings of the Second Sussex Conference, 1977, Lecture Notes in Mathematics 722, Springer-Verlag, Berlin (1979)

[5] T Kanenobu, The augmentation subgroup of a pretzel link, Mathematics Seminar Notes, Kobe University, 7 (1979) 363-384

[6] J D Masters, Virtual homology of surgered torus bundles", to appear in Pacific J. Math.

[7] W D Neumann, A W Reid, Arithmetic of hyperbolic 3-manifolds, Topology '90, de Gruyter (1992) 273-309

[8] J-P Otal, Thurston's hyperbolization of Haken manifolds, from: "Surveys in Differential Geometry, Vol. III", (Cambridge MA 1996), Int. Press, Boston MA (1998) 77-194

[9] A W Reid, Arithmeticity of knot complements, J. London Math. Soc. 43 (1991) $171-184$

[10] A W Reid, Isospectrality and commensurability of arithmetic hyperbolic 2-and 3-manifolds, Duke Math. J. 65 (1992), no. 2, 215-228

[11] R Riley, Parabolic representations and symmetries of the knot $9_{32}$, from: "Computers and Geometry and Topology", (M C Tangora, editor), Lecture Notes in Pure and Applied Math. 114, Dekker (1988) 297-313

[12] W Thurston, A norm for the homology of 3-manifolds, Mem. Amer. Math. Soc. 59 (1986) 99-130

[13] F Waldhausen, On irreducible 3-manifolds which are sufficiently large, Ann. of Math. 87 (1968) 195-203 